\title{
A CHARACTERIZATION OF HEREDITARILY INDECOMPOSABLE CONTINUA
}

\section{ALBERT L. CRAWFORD AND JOHN JOBE}

ABSTRACT. In this paper a characterization of a hereditarily indecomposable continuum is stated and proved. The motivation for this characterization is a theorem in a recent article by John Jobe.

In this paper a characterization of a hereditarily indecomposable continuum is stated and proved. The motivation for this characterization is a theorem proved by Jobe in [1]. This result is as follows:

THEOREM 1. If $M$ is the 2-finished sum of compact continua, $M_{1}$ and $M_{2}$, such that $M_{1}$ is hereditarily indecomposable and $M_{1} \cap M_{2} \neq \varnothing$, then there exists at least one point in $M_{1} \cap M_{2}$ which is a limit point of both $\left(M_{1}-M_{2}\right)$ and $\left(M_{2}-M_{1}\right)$.

Definition. The set $M$ is the 2 -finished sum of continua $M_{1}$ and $M_{2}$ if $M=M_{1} \cup M_{2}$ and $M_{1}-M_{2} \neq \varnothing$ and $M_{2}-M_{1} \neq \varnothing$.

We shall consider the space $S$ to be a Moore space satisfying Axiom 0 and Axiom 1 of R. L. Moore.

First, we suspected that the hypothesis in Theorem 1 was too

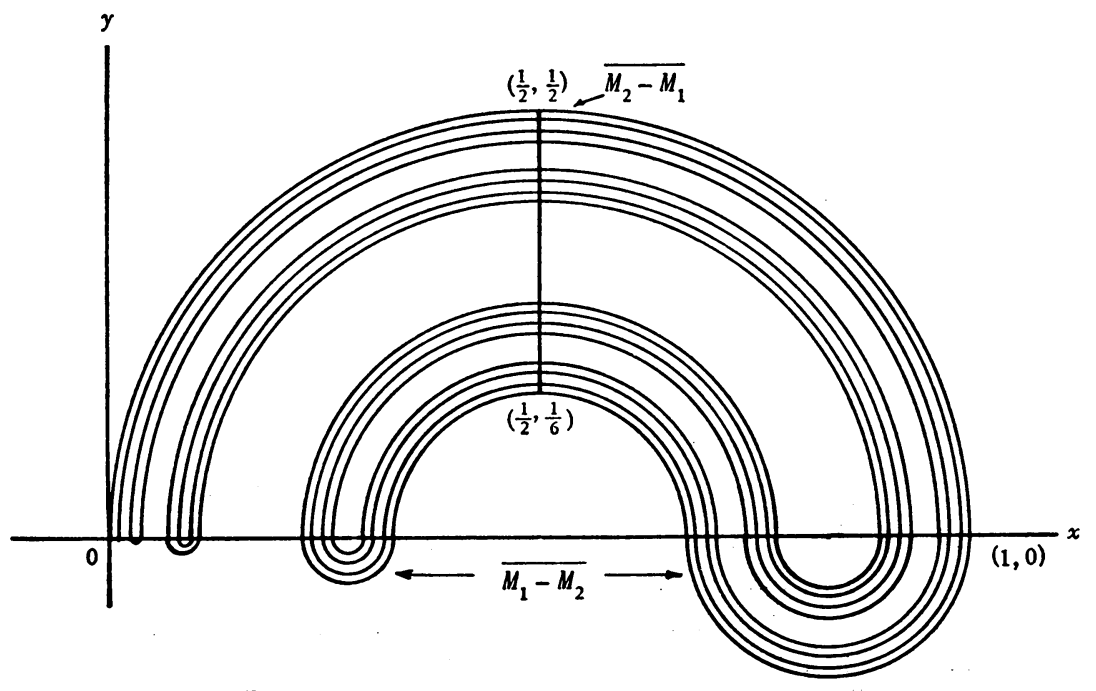

Received by the editors February 19, 1970.

AMS 1969 subject classifications. Primary 5438; Secondary 5420.

Key words and phrases. Hereditarily indecomposable continuum, pseudo-arc, Brouwer continuum. 
strong. That is, we suspected that $M_{1}$ need only be an indecomposable continuum rather than hereditarily indecomposable. The following example in the plane emphasizes the importance of the hypothesis of Theorem 1 as stated. It shows the existence of a compact indecomposable continuum $M_{1}$ and a compact continuum $M_{2}$ satisfying the hypothesis of Theorem 1 with the exception that $M_{1}$ is not hereditarily indecomposable and the conclusion of Theorem 1 is not true.

EXAMPLE 1. Let $M_{1}$ be the indecomposable continuum in the plane consisting of those semicircles lying above the $x$-axis centered at $(1 / 2,0)$ with endpoints in the Cantor ternary set on the $x$-axis together with those semicircles lying below the $x$-axis with centers at $\left(1 /\left(6 \cdot 3^{i}\right), 0\right)$ and endpoints in the Cantor set. See [3].

Let $M_{2}$ be the continuum consisting of $M_{1}$ intersected with the closed upper-half plane together with the line segment from $(1 / 2,1 / 6)$ to $(1 / 2,1 / 2)$.

Then $M_{1}-M_{2}$ lies below the $x$-axis and $M_{2}-M_{1}$ lies above the line $y=1 / 6$. Hence there exists no point that is a limit point of both $M_{1}-M_{2}$ and $M_{2}-M_{1}$.

The characterization of a hereditarily indecomposable continuum is in terms of the following defined Property $Q$. The definition of Property $Q$ is motivated by the condition in Theorem 1.

Definition. Let $S$ be a Moore space and $M$ a continuum in $S$. Then $M$ has Property $Q$ in $S$ if and only if for every compact continuum $N$ in $S$ such that $N \cap M \neq \varnothing$ and $N \cup M$ is the 2-finished sum of $N$ and $M$, then there exists a point $p \in M \cap N$ such that $p$ is a limit point of both $M-N$ and $N-M$. A compact continuum $M$ in $S$ has Property $Q$ hereditarily in $S$ if and only if each subcontinuum of $M$ has Property $Q$ in $S$.

THEOREM 2. Let $T$ be a Moore space and $M$ a compact continuum in $T$. Then $M$ is hereditarily indecomposable if and only if for every function $f$ and Moore space $S$ such that $f$ imbeds $M$ in $S$, then $f(M)$ has Property $Q$ hereditarily in $S$.

Proof. Assume the condition of the theorem. Let $M$ be a compact continuum in a Moore space $S$ and suppose that $M$ is not hereditarily indecomposable. Then there exists a decomposable subcontinuum $M^{\prime}=H \cup K \subset M$ where $H$ and $K$ are proper subcontinua of $M^{\prime}$ and $h \in H-K$. Note that since $S$ is a Moore space then $S \times S$ is also a Moore space. Define maps $f$ and $g$ from $M$ to $S \times S$ as $f(m)=(m, h)$ and $g(m)=(h, m)$ for each $m \in M$. Then, since both $f$ and $g$ imbed $M$ in $S \times S, f\left(M^{\prime}\right)=M^{\prime} \times\{h\}=M_{1}$ and $g\left(M^{\prime}\right)=\{h\} \times M^{\prime}=M_{2}$ are homeomorphic to $M^{\prime}$. Let $H_{1}=f(H)$ and $K_{1}=f(K)$. Then $M_{1}=H_{1}$ $\cup K_{1} \subset f(M)$ is decomposable with $(h, h) \in H_{1}-K_{1}$ since $h \in H-K$. 
Also the definitions of $f$ and $g$ imply that $M_{1} \cap M_{2}=\{(h, h)\}=H_{1} \cap M_{2}$ since $H_{1} \subset M^{\prime}$. Thus $H_{1} \cup M_{2}$ is a continuum in $S \times S$. Furthermore, $\left(H_{1} \cup M_{2}\right)-M_{1}=M_{2}-H_{1}$, and hence

$$
\overline{\left(H_{1} \cup M_{2}\right)-\bar{M}} \subset M_{2} \text {. }
$$

Also $M_{1}-\left(H_{1} \cup M_{2}\right)=M_{1}-H_{1} \subset K_{1}$ and

$$
\overline{M_{1}-\left(H_{1} \cup \overline{\left.M_{2}\right)}\right.} \subset K_{1} \text {. }
$$

Since $K_{1}$ and $M_{2}$ are disjoint closed sets, no point is a limit point of both $M_{1}-\left(H_{1} \cup M_{2}\right)$ and $\left(H_{1} \cup M_{2}\right)-M_{1}$. Hence, $M_{1}=f\left(M^{\prime}\right)$ does not have Property $\mathrm{Q}$ in $S \times S$. Therefore, $f(M)$ does not have Property $\mathrm{Q}$ hereditarily in $S \times S$ which is a contradiction. Therefore, $M$ is hereditarily indecomposable.

Conversely assume that $M$ is a compact hereditarily indecomposable continuum in a Moore space $T$. Let $f$ be any function that imbeds $M$ in a Moore space $S$ and consider $f(M)$. Since each subcontinuum of $f(M)$ is itself hereditarily indecomposable, applying Theorem 1 we see that each subcontinuum of $f(M)$ has Property $Q$ in $S$. Hence $f(M)$ has Property $Q$ hereditarily in $S$ and the condition of the theorem follows.

We thought that in Theorem 2 the condition "for every function $f$ and Moore space $S$ such that $f$ imbeds $M$ in $S, f(M)$ has Property $Q$ hereditarily in $S$ " could be replaced by the condition " $M$ has Property $Q$ hereditarily in $T$." To see that this cannot be done the following example exhibits a Moore space $T$ and a decomposable compact continuum $M$ in $T$ such that $M$ has Property $Q$ hereditarily in $T$. Thus, this example complements the statement of Theorem 2 .

Example 2. Let $S_{1}$ and $S_{2}$ be two pseudo-arcs in the plane constructed from $(-1,0)$ to $(0,0)$ and $(0,0)$ to $(1,0)$ respectively such that $S_{1} \cap S_{2}=\{(0,0)\}$. Let $p=(0,0)$. Let $T$ be the subspace of the plane such that $T=S_{1} \cup S_{2}$. Let $H$ and $K$ be nondegenerate proper subcontinua of $S_{1}$ and $S_{2}$ respectively such that $H \cap K=\{p\}$. Then $M=H \cup K$ is a decomposable compact continuum that has Property $Q$ hereditarily in $T$.

Suppose $N$ is a subcontinuum of $T$ such that $N \cap M \neq \varnothing$ and $N \cup M$ is the 2-finished sum of $N$ and $M$. Clearly $p \in N$ and we may assume that $\left(N \cap S_{1}\right) \subset H$ and $K \subset\left(N \cap S_{2}\right)$. Then $M-N=H-\left(N \cap S_{1}\right)$ and $N-M=\left(N \cap S_{2}\right)-K$. Because $H$ and $N \cap S_{2}$ are pseudo-arcs, $p$ is a limit point of both $H-\left(N \cap S_{1}\right)$ and $\left(N \cap S_{2}\right)-K$. Since $p \in M \cap N$ it has been verified that $M$ has Property $Q$ in $T$.

Now if $M^{\prime}$ is a subcontinuum of $M$, then either (a) $M^{\prime} \subset S_{1}$ or (b) $M^{\prime} \subset S_{2}$ or (c) $M^{\prime}-S_{1} \neq \varnothing$ and $M^{\prime}-S_{2} \neq \varnothing$. In cases (a) and (b), $M^{\prime}$ 
is hereditarily indecomposable and hence has Property $Q$ in $T$. In case (c), $M^{\prime}$ has Property $Q$ in $T$ by the method used to show that $M$ has Property $Q$ in $T$. Therefore, $M$ has Property $Q$ hereditarily in $T$.

Since $M$ is decomposable the example is verified.

There is a natural question suggested by Theorem 2 and Example 2-namely, is the property in question really a property of the embedding or a property of the containing space? More precisely, if $M$ is a compact continuum, $S$ is a Moore space, and $f$ and $g$ are two embeddings of $M$ in $S$, does $f(M)$ have Property $Q$ hereditarily in $S$ if and only if $g(M)$ has Property $Q$ hereditarily in $S$ ? Example 3 gives a negative answer to this question.

Example 3. Let $T$ and $M$ be as defined in Example 2. By Theorem 2 there exists a Moore space $T_{1}$ and an imbedding function $f$ from $M$ to $T_{1}$ such that $f(M)$ does not have Property $Q$ hereditarily in $T_{1}$. The space $T_{1}$ can be picked such that $T \cap T_{1}=\varnothing$. Let $g$ be the imbedding from $M$ to $T$ such that $g(m)=m, m \in M$. Example 2 reveals that $g(M)$ has Property $Q$ hereditarily in $T$.

Let $S=T \cup T_{1}$ and $A$ be open in $S$ if and only if $A$ is the union of an open set in $T$ and an open set in $T_{1}$. Note that $S$ is a Moore space. It follows that $f$ and $g$ can be thought of as imbeddings of $M$ into $S$ and clearly $g(M)$ has Property $Q$ hereditarily in $S$ while $f(M)$ does not have Property $Q$ hereditarily in $S$.

The authors have been able to find two other characterizations of hereditarily indecomposable continua. These two can be found in [2] and [4].

\section{BiBLIOGRAPHY}

1. John Jobe, The intersection of indecomposable continua, Proc. Amer. Math. Soc. 23 (1969), 623-624. MR 40 \#877.

2. J. L. Kelley, Hyperspaces of a continuum, Trans. Amer. Math. Soc. 52 (1942), 22-36. MR 3, 315.

3. K. Kuratowski, Topologie. Vol. 2, Monografie Mat., Tom 21, PWN, Warsaw, 1961; English transl., Academic Press, New York; PWN, Warsaw, 1969. MR 24 \#A2958.

4. William R. Zame, A characterization of hereditarily indecomposable continua, Proc. Amer. Math. Soc. 17 (1966), 709-710. MR 33 \#3274.

Oklahoma State University, Stillwater, OkLahoma 74074 